\title{
DISCOVERY OF X-RAY COUNTERPARTS TO GAMMA RAY BURSTS BY BEPPOSAX
}

\author{
L. PIRO \\ Istituto Astrofisica Spaziale,.C.N.R. \\ Via Fosso del Cavaliere, 00131 Roma, Italy \\ ON BEHALF OF THE BEPPOSAX TEAM
}

\section{Introduction}

The nature of Gamma-Ray Burst (GRB) has been the object of many investigations but their origin has remained a mistery primarily for the difficulties in finding a counterpart. This difficulty derived from the intrinsically poor positioning capability of available GRB detectors.

In the first part of 1997 BeppoSAX has provided the real breakthrough in this domain. BeppoSAX payload is a combination of different instruments (Piro et al. 1995; Boella et al. 1997). While the NFI telescopes (Parmar et al. 1997; Boella et al. 1997b) probe a narrow field with high sensitivity, performing its observation plan as any regular observatory, the two Wide Field Cameras (40 x $40 \circ$ at zero response) (Jager et al. 1997) are continuously monitoring in the $2-30 \mathrm{keV}$ band, with good time and energy resolution, two other regions of the sky. The Gamma-Ray Burst Monitor (Frontera et al. 1997) electronics provide an onboard trigger on suspect GRBs and high temporal resolution recording of data under these trigger conditions. The combination of these two was very effective. On the GRBM trigger time the Science Operation Center looks for an excess in the WFCs ratemeters. If this is found an image is made at time corresponding to this excess. The burst appears as a point source right at that time while other excesses such as scattered solar flare or magnetospheric events is cancelled by the coded mask image reconstruction process.

The peculiarity of having all the instruments onboard BeppoSAX under the same scientific and operation control has allowed for the development of 
a fast follow-up pointing of the NFIs in the case of serendipitous occurrence of a GRB in the field of view of one of the two Wide Field Cameras.

In these cases BeppoSAX can provide 1) a first light curve starting from $10 \mathrm{~s}$ before the trigger in the band $40-700 \mathrm{keV}$. 2) An X-ray light curve $(2-30 \mathrm{keV})$ from long time before the GRB, during it and after, only limited by the sensitivity to the reduced fluxes of the GRB. 3) A light curve with the Narrow Field Instruments of the afterglow source from about 6 hours until it has decayed to levels of the order of $310^{-14} \mathrm{erg} / \mathrm{cm}^{2} \mathrm{~s}$.

\section{X-ray afterglows of GRB}

The first detection of an X-ray counterpart to a GRB (hereafter afterglow happened on Feb. 28, 1997 (Costa et al. 1997). Fig. 1 shows the images recorded by the MECS in the $2-10 \mathrm{keV}$ range 8 hours and 3 days after the GRB. The previously unknown X-ray source lying in the 3 arcmin WFC error circle decreased by a factor of 20 . The source was observed to decay already during the first pointing (Fig.2) and continue to decrease after the second BeppoSAX observation, as recorded by ASCA (Yoshida et al. 1997) and then ROSAT (Frontera et al. 1997b). These (2-10 keV) data are well reproduced by a power law with a slope of 1.3. By extrapolating this curve to the time of the GRB we found a value surprisingly consistent with the flux of a train of pulses following the main event. A similar behaviour was then observed in the second afterglow discovered by BeppoSAX in April 97 in GB970402 (Piro et al. 1997).

In the case of GB970111 (Butler et al. 1997, Feroci et al. 1997) we found a marginal detection at a flux much below one would have expected extrapolating the evolution of the February and April events.

Light curve of GRB970508 (Piro et al. 1997b) is less regular. During the first pointing (after 6 hours) the source was varying but not with a

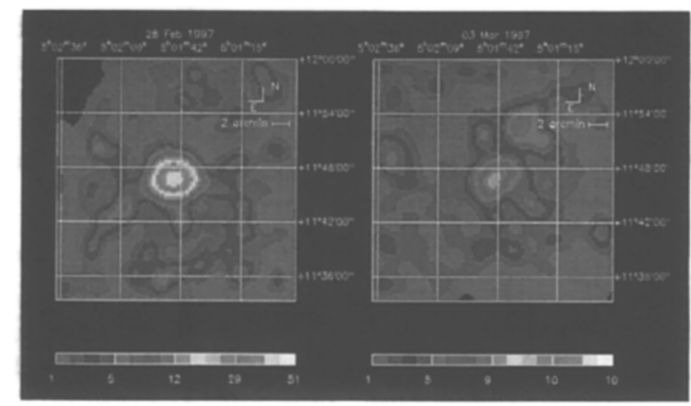

Figure 1. GB970228: MECS images of the afterglow observed on Feb.28, 8 hours after the burst (left image). On Mar.3 (right image) the source had decreased by a factor of 20 
monotonic decay. In subsequent pointing, as shown in fig.3, it decayed with a light curve very far from a power law. This could suggest the existence of a gap between the GRB and its afterglow and possibly a behaviour similar to that of the optical counterpart that peaked two days after the GRB. But in this case data from Wide Field Camera after the initial burst show that a faint afterglow source is present at levels that can be reasonably connected with those of the subsequent NFI observations.

In the present very incomplete picture it seems more likely that the power law is an average trend, with a slope that can be substantially different in each GRB, and the actual light curve can include significant flare activity around this trend.

TABLE 1. Simultaneous detection of GRB's with the BeppoSAX GRBM and WFC and follow up with NFI

\begin{tabular}{lllll}
\hline GRB & $\begin{array}{l}\text { peak flux } \\
(40-700 \mathrm{keV})\end{array}$ & $\begin{array}{l}\text { peak flux } \\
(2-26 \mathrm{keV})\end{array}$ & $\begin{array}{l}\mathrm{TOO}^{2} \\
\Delta \mathrm{T}\end{array}$ & $\begin{array}{l}\text { TOO result } \\
\mathrm{F}(2-10 \mathrm{keV})\end{array}$ \\
\hline $960720^{1}$ & $1.0 \times 10^{-6}$ & $2.5 \times 10^{-8}$ & 1038 & $1.0 \times 10^{-13}$ QSO 4C49.29 \\
970111 & $4.5 \times 10^{-6}$ & $1.4 \times 10^{-7}$ & 16 & $\leq 5 \times 10^{-14}$ \\
970228 & $3.0 \times 10^{-6}$ & $1.4 \times 10^{-7}$ & 8 & $3 \times 10^{-12} 1 \mathrm{SAXJ} 0501.7+1146$ \\
970402 & $2.5 \times 10^{-7}$ & $1.6 \times 10^{-8}$ & 8 & $2 \times 10^{-13} 1 \mathrm{SAXJ1450.1-6920}$ \\
970508 & $3.5 \times 10^{-7}$ & $6 \times 10^{-8}$ & 6 & $8 \times 10^{-13} 1 \mathrm{SAXJ} 0653.8+7916$ \\
\hline
\end{tabular}

Note: fluxes in cgs units; ${ }^{1}$ : found during off-line analysis (Piro et al. 1997c) ${ }^{2}$ : TOO start in hrs after the GRB trigger

\section{References}

Boella G. et al., A\&A Supp. Ser., 122, 299-307 (1997)

Boella, G., Chiappetti, L. et al., 1997b, A\&AS, 122, 327

Butler, R.C., et al. 1997, IAU Circular n. 6539.

Costa, E. et al. 1997, Nature, 387, 783

Jager, R., et al. 1997, A\&A Suppl. Ser., 125, 557

F. Frontera, et al., A\&A Supp. Ser., 122, 357-369 (1997)

Frontera et al. 1997b, IAUC 6637

Parmar A.N. et al. 1997, A\&AS, 122, 309

Piro L., Scarsi L. \& Butler R.C. 1995 SPIE 2517, 169

Piro, L. et al. 1997, IAU Circ. 6617

Piro, L. et al. 1997b, A\&AL, in press (astro-ph/9710355)

Piro et al. 1997c, A\&A, in press (astro-ph/9707215)

Yoshida A et al. 1997 IAUC 6593 


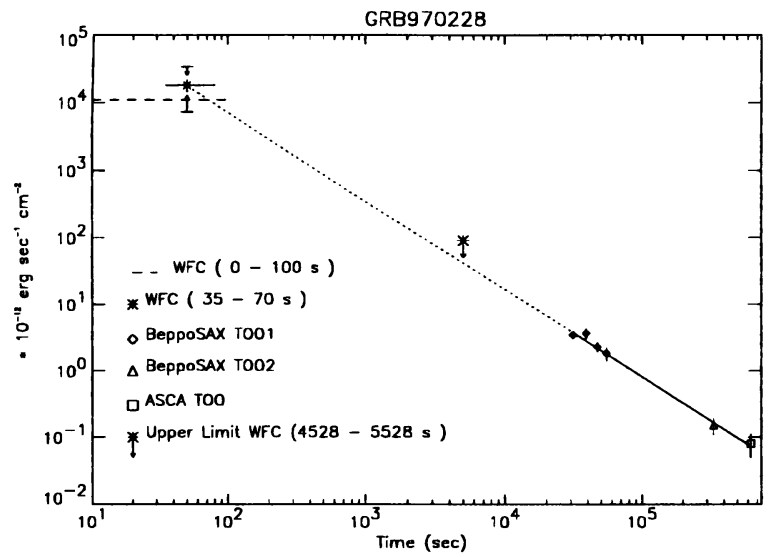

Figure 2. GB970228: X-ray evolution of the GRB from the initial event to its afterglow

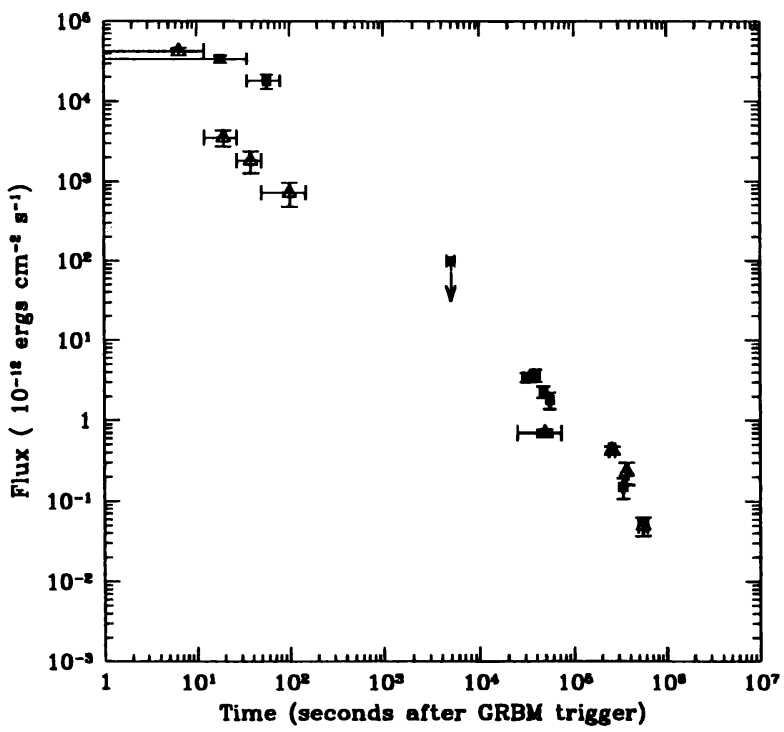

Figure 3. GRB970228 X-ray light curves from BeppoSAX Wide Field Cameras and Narrow Field Instruments of GRB970228 (squares) and GRB970508 (triangles). For the first part of the burst the average flux is displayed 